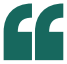

Our expectations derived from simple textbook electron donation/ withdrawal considerations were turned on their head

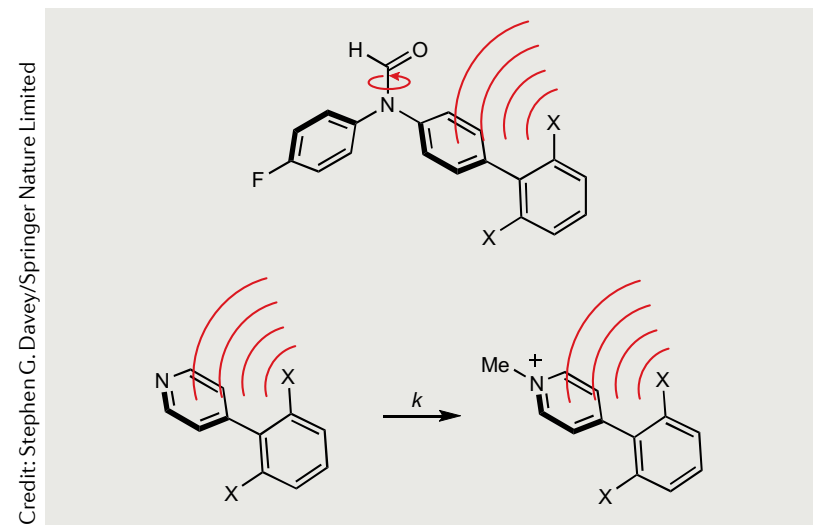

ELECTRONIC INTERACTIONS

\section{Feeling the effects}

When studying reaction mechanisms, the effects of small changes in substrate structure on reaction outcome and rate are often rationalized by considering the electron-donating or electron-withdrawing properties of a particular substituent. Indeed, these terms are a key component of undergraduate teaching in organic chemistry. They are usually considered almost entirely as being through-bond effects, but recently questions have been raised about the relative importance of simple through-space interactions. Now, Scott Cockroft and co-workers from the University of Edinburgh have built a series of molecules that enable the direct experimental study of through-space effects and reached some surprising conclusions.

"In 2008, Wheeler and Houk reported a seminal computational study on the interactions between substituted benzenes," explains Cockroft. "They showed that the sum of isolated electrostatic potentials of the ring and substituents provided a remarkably good approximation of the interactions and thus suggested that through-space interactions had thought. We were keen to see were far more important than many whether we could reproduce this in an experimental model in the presence of a solvent."

Cockroft and co-workers studied a series of 25 'molecular balances' in which the electronic effect of substituents can be measured by its influence on the conformational equilibrium of a nearby formamide. A substituent inducing a positive electrostatic potential would favour a conformation in which the electron-rich oxygen atom of the carbonyl group was in closest proximity to the substituent, while a substituent inducing a negative electrostatic potential would repel the oxygen. Similarly, they studied the $N$-methylation reactivity of a series of 17 phenylpyridine molecules. "It is a challenge to design a system in which through-bond influences are removed - there are always bonds between the substituent and the site where its effects are manifested," explains Cockroft. "We reasoned that a biaryl system would allow us to have substituents pointed 'backwards' towards the site where the effect was measured and this would help minimize through-bond effects and thus ensure that any measured effect would be largely the result of through-space electrostatics."

Cockroft admits to being sceptical of the Wheeler-Houk model but was rapidly convinced by the data they were able to collect. "Our expectations derived from simple textbook electron donation/ withdrawal considerations were turned on their head," he explains. They found, for example, that a nitro group - usually considered to be strongly electron withdrawing could exert an influence similar to that of an electron-donating group when it was oriented to point backwards over a biaryl ring. Similarly, the classical electron-donating influence of methoxy or hydroxyl substituents could be switched off, or even inverted by twisting the orientation of the substituent. "These observations can only be rationalized if through-space electrostatic field effects dominate over through-bond contributions," he continues.

Terms such as 'electron donating' and 'electron withdrawing' have proved highly useful in teaching the basic principles of organic chemistry, but this work shows that they can often be mechanistically misleading when going beyond simple textbook examples. "These terms should be strictly reserved for true delocalization influences such as resonance and hyperconjugation, but even then we must consider, for example, that much of the influence of a nitro group is manifested via electric fields, not by electron delocalization," says Cockroft.

Beyond just improving mechanistic understanding, however, Cockroft and co-workers see real applications of these effects. "The through-space effects of two nearby methoxy groups can have a larger overall electronic effect than a conjugated nitrogen lone-pair without any attendant problem of side reactions," he says. "One might imagine such effects being exploited in ligand design for catalysis."

Stephen G. Davey

ORIGINAL ARTICLE Burns, R. J. et al. Quantifying through-space substituent effects. Angew. Chem. Int. Ed. https://doi.org/10.1002/anie.202006943 (2020)

RELATED ARTICLE Wheeler, S. E. \& Houk, K. N. Substituent effects in the benzene dimer are due to direct interactions of the substituents with the unsubstituted benzene. J. Am. Chem. Soc. 130 10854-10855 (2008) 